\section{Adjuvante Chemotherapie bei Darmkrebs im Stadium II: Wichtiger als oft angenommen}

\author{
Internationale Leitlinien empfehlen in sehr unterschiedlichem Maße den \\ Einsatz einer adjuvanten Chemotherapie bei einem Kolonkarzinom im UICC- \\ Stadium II. In Deutschland wird hierzu derzeit eine "Kann"-Empfehlung \\ ausgesprochen. Der Nutzen könnte aber größer sein als angenommen.
}

\footnotetext{
$\mathrm{D}$ arauf weist die retrospektive Analyse einer Kohorte von 153.110 Patienten mit einem solchen Kolonkarzinom aus der US-amerikanischen nationalen Krebsdatenbank hin. Alle Patienten hatten ihre Diagnose zwischen 1998 und 2006 erhalten und waren primär operativ behandelt worden. Für alle Patienten, die in die Auswertung eingingen, mussten Angaben zu Demografie, Tumorcharakteristika, Therapiemanagement und Überleben bis zum Jahr 2011 zur Verfügung stehen. Jeder 5. Patient (19,3\% der Patienten mit niedrigem und $21,7 \%$ der Patienten mit hohem Risiko) hatte eine adjuvante Chemotherapie erhalten, häufiger war dies u. a. bei jüngeren Patienten
} Wirkstoffen zu. einer T4-Histologie, aber auch unabhängig vom Alter oder der Art des Chemotherapieregimes zu finden. Das blieb auch nach Berücksichtigung weiterer Einflussfaktoren und bei einer Propensity-Score-Gewichtung so, mit einer Hazard Ratio von 0,76 ( $\mathrm{p}<0,001)$. Bemerkenswert war, dass es keinen Unterschied machte, ob nur mit Fluoropyrimidin oder mit mehreren Agenzien behandelt worden war.

(<65 Jahre) und Männern sowie Patien ten ohne Komorbiditäten der Fall. Vor dem Jahr 2004 überwogen Therapien mit 5-Fluorouracil (5-FU) mit oder ohne Folinsäure. Danach nahm die Anwendung von Therapieschemata mit mehreren

Die adjuvante Chemotherapie war assoziiert mit einer Verlängerung des medianen Gesamtüberlebens (OS) von 7,0 auf 13,2 Jahre. Der OS-Vorteil war in allen analysierten Subgruppen unabhängig von Hochrisikomarkern der Erkrankung wie einer ungünstigen oder schlecht differenzierten Histologie, weniger als 12 untersuchten Lymphknoten, positiven Resektionsrändern oder
Fazit: In dieser großen retrospektiven Analyse ließ sich ein deutlicher OS-Vorteil durch eine adjuvante Chemotherapie bei Patienten mit Kolonkarzinom im Stadium II feststellen. Der Vorteil war dabei unabhängig von der Pathologie des Tumors, dem Alter des Patienten oder dem eingesetzten Regime. Prospektiv erhobene Daten sind wünschenswert, um die Rolle der Adjuvans in diesem Patientenkollektiv zu sichern.

Friederike Klein

Casadaban L et al. Adjuvant chemotherapy is associated with improved survival in patients with stage II colon cancer. Cancer. 2016; 122(21):3277-87.

\title{
mCRC mit RAS-Wildtyp: Biologische Therapien im Meta-Vergleich
}

\begin{abstract}
Direkte Vergleichsstudien von Inhibitoren des EGFR und des VEGF zusätzlich zu einer Erstlinienchemotherapie des metastasierten kolorektalen Karzinoms (mCRC) vom RAS-Wildtyp haben unterschiedliche Ergebnisse erbracht. In einer Metaanalyse wurde nun der Einfluss beider Strategien auf das Gesamtüberleben verglichen.
\end{abstract}

\footnotetext{
Ü ber PubMed identifizierten die Forscher 3 Studien zur Erstlinientherapie des metastasierten Kolorektalkarzinoms (mCRC), die EGFR(,epidermal growth factor receptor")-Inhibitoren (EGFRI; Cetuximab oder Panitumomab) plus Chemotherapie und einen VEGF („vascular endothelial growth factor")-Inhibitor (Bevacizumab) plus Chemotherapie verglichen hatten, und aktualisierten die Studienergebnisse anhand jüngerer Kongresspräsentationen und noch nicht publizierter Daten, soweit verfügbar.

Die Auswertung der Daten von 1.096 Patienten mit mCRC und RAS-Wildtyp zeigte einen Vorteil für EGFRI plus Che-
}

motherapie gegenüber Bevacizumab plus Chemotherapie hinsichtlich des Gesamtüberlebens (OS; Hazard Ratio [HR] 0,80; $95 \%$-Konfidenzintervall [95\%-KI] $0,68-0,93)$ sowie der objektiven Ansprechrate (ORR; Odds Ratio [OR] 0,57, 95\%-KI 0,42-0,76). Kein Unterschied fanden die Forscher dagegen hinsichtlich des progressionsfreien Überlebens (PFS; HR 0,98, 95\%-KI 0,86-1,12) und der Resektionsrate (OR 0,93, 95\%-KI 0,57-1,51). Als explorativen Endpunkt analysierten sie auch den Anteil der Patienten mit einem frühen Tumorschrumpfen (ETS) - definiert als Reduktion um mindestens $20 \%$ in Woche 8 . Auch hier zeigte sich ein Vorteil für die
Therapie mit EGFRI plus Chemotherapie (OR 0,48, 95\%-KI 0,33-0,71).

Eine Metaanalyse zu Sicherheitsaspekten konnte aufgrund fehlender Daten nicht vorgenommen werden, wäre aber im Hinblick auf die Bewertung beider Therapieoptionen für mCRC-Patienten mit RAS-Wildtyp wichtig, betonen die Forscher.

Fazit: Die Metaanalyse auf Studienebene weist auf einen möglichen Vorteil der Erstlinientherapie mit EGFRI plus Chemotherapie gegenüber Bevacizumab plus Chemotherapie für das OS, die ORR und das ETS beim mCRC mit RAS-Wildtyp hin. Die Forscher regen eine Metaanalyse auf Patientenebene an. Friederike Klein

Heinemann V et al. A study-level meta-analysis of efficacy data from head-to-head first-line trials of epidermal growth factor receptor inhibitors versus bevacizumab in patients with RAS wild-type metastatic colorectal cancer. Eur J Cancer. 2016;67:11-20. 